\title{
METODOLOGÍAS PARA LA ESTIMACIÓN DEL COEFICIENTE DE ESCORRENTÍA EN ÁREAS URBANIZADAS MEDIANTE TELEDETECCIÓN
}

\author{
Eugenia Chiarito $^{(1)}$; Erik Zimmermann ${ }^{(1)(2)}{ }^{(*)}$ y Soledad Méndez Zacarías ${ }^{(1)}$ \\ (1) Centro Universitario Rosario de Investigaciones Hidroambientales, Facultad de Ciencias Exactas, \\ Ingeniería y Agrimensura, Universidad Nacional de Rosario, Rosario, Santa Fe, Argentina. \\ ${ }^{(2)}$ CONICET Consejo Nacional de Investigaciones Científicas y Técnicas. Argentina. \\ ${ }^{(*)}$ e_mail: erikz@fceia.unr.edu.ar
}

\begin{abstract}
RESUMEN
El coeficiente de escorrentía (C) del método racional, correspondiente a un área determinada, se estima en base al uso y el tipo de suelo existentes, pautado por una vasta bibliografía internacional. Convencionalmente esta clasificación de usos de suelo se realiza a través de la interpretación de imágenes satelitales y de su constatación en campo. Este trabajo se centra en la comparación entre la metodología convencional y dos metodologías que permiten vincular valores de $\mathrm{C}$ con usos de suelos obtenidos mediante técnicas de Teledetección. En una de ellas se busca esta correlación a partir del uso de índices normalizados de construcción (NDBI y UI), mientras que en la otra se determinan las coberturas a partir de una clasificación supervisada. En ambos casos se utilizan imágenes del satélite LANDSAT 8. Las metodologías fueron validadas contrastando resultados en 3 cuencas urbanas de la ciudad de Rosario, Santa Fe. Con ambas se obtuvieron resultados con diferencias respecto a la metodología convencional comprendidas entre el $3 \%$ y el $16 \%$. Estas propuestas alternativas implican una reducción significativa del tiempo requerido para la estimación del C.
\end{abstract}

Palabras clave: uso de suelo, coeficiente de escorrentía, teledetección.

\begin{abstract}
The estimation of the runoff coefficient (C) of the rational method in a given area is based on the land use and land cover based on a wide international bibliography. Conventionally this classification is done by interpreting satellite images supported with field supervision. This work focuses on the comparison between the conventional methodology and two methodologies that relate $\mathrm{C}$ values with land use estimation based on remote sensing techniques. One of them is based on standardized building indexes (NDBI and UI), while the other is based on supervised classification. In both cases, LANDSAT 8 images are processed. The methodologies were validated by contrasting results in 3 urban basins of Rosario city, in Santa Fe. Differences between $3 \%$ and $16 \%$ were obtained in both cases when compared to the conventional methodology These alternative proposals imply a significant reduction in the time demanding for the $\mathrm{C}$ estimation.
\end{abstract}

Keywords: land use; runoff coefficient, remote sensing. 


\section{INTRODUCCIÓN}

El escurrimiento en cuencas urbanas se puede dividir en dos componentes interrelacionados, los cuales, en función de la magnitud de los eventos de lluvia, presentarán funcionamientos hidráulicos diferentes.

Un componente es el escurrimiento superficial por techos, veredas, calles, playas de estacionamiento, cunetas, zanjas, etc. El mismo puede drenar hacia la red de conductos a través de obras de captación, hacia canales urbanos secundarios o directamente hacia el cuerpo receptor principal (río, mar, lago, etc.). El otro componente es el escurrimiento en la red de conductos, los cuales transportan el agua captada del sistema superficial por medio de bocas de tormenta, captaciones de zanja, etc. Los conductos drenan hacia el cuerpo receptor principal o en ocasiones hacia canales urbanos secundarios. La capacidad de descarga de la red de conductos se establece a partir de lluvias de diseño máximas de una duración determinada y valores bajos de períodos medios de retorno, generalmente de 2 a 5 años. La adopción de recurrencias bajas obedece a razones de orden económico como así también a las limitaciones de espacio físico que plantea la realidad urbana. De cualquier modo es esperable que, para un sistema bien diseñado, durante tales eventos no se produzcan sustanciales inundaciones en la ciudad, y para tormentas de recurrencia mayor, dicho sistema permita mitigar en algún grado los posibles anegamientos.

El proyecto del sistema de una red de drenaje implica la selección del diámetro de los conductos, las pendientes, la distribución y localización de los conductos, de las bocas de tormenta, de las bocas de registro, etc.

Una vez que se ha seleccionado una distribución para los conductos colectores, se puede utilizar el método racional para determinar el caudal máximo de diseño. Con dicho caudal podrán establecerse las dimensiones de las bocas de tormenta, el diámetro de los conductos, las dimensiones de eventuales canales, etc. El método racional, a pesar de las críticas, ha sido y es actualmente aplicado dada su simplicidad y facilidad de implementación. Una de las implicancias de mayor incertidumbre en el método es el denominado coeficiente de escorrentía C. Normalmente se recurre a tablas, publicadas en la bibliografía internacional, donde el valor de $\mathrm{C}$ queda definido en función de las características del sistema, afectado por una ponderación areal según cada configuración presente en el área de interés.

En este sentido, intenta hacer un aporte el siguiente trabajo, analizando distintas metodologías basadas en técnicas de teledetección, con la intención de sistematizar la estimación de este parámetro. Las metodologías propuestas se comparan entre sí y con los valores obtenidos de la manera convencional en tres cuencas urbanas de la ciudad de Rosario (Santa Fe, Argentina).

\section{EL MÉTODO RACIONAL}

El método racional fue desarrollado por Mulvaney (1850) cuya propuesta se basa en que: dada una cuenca de área A con un tiempo de concentración $\mathrm{Tc}$, si se produce una lluvia $\mathrm{P}$ con duración $\mathrm{D}=\mathrm{Tc}$, el caudal generado en el punto de salida será el máximo, ya que estará aportando toda la cuenca.

El método plantea una proporcionalidad entre el caudal máximo y la intensidad de lluvia y puede expresarse como:

$\mathrm{Q}=\mathrm{C}_{\mathrm{u}} \cdot$ C.i.A

Donde: $Q$ es el caudal de diseño $\left(\mathrm{L}^{3} / \mathrm{T}\right), \mathrm{C}_{\mathrm{u}}$ es el coeficiente de conversión de unidades, $\mathrm{C}$ es el coeficiente de escurrimiento (adimensional), i es la intensidad de la precipitación de diseño (L/T), y A es el área de drenaje de cuencas $\left(\mathrm{L}^{2}\right)$.

El coeficiente de escurrimiento $C$, es la variable que presenta mayor incertidumbre en su determinación, y representa una relación adimensional entre la lámina de escorrentía superficial generada por una determinada cuenca y la lámina de la precipitación. El coeficiente de escorrentía varía con la pendiente, condiciones de la superficie y de la cubierta vegetal y el tipo de suelo hidrológico. Las superficies que son relativamente impermeables como las calles y plazas de aparcamiento tienen coeficientes de escorrentía que se aproximan a uno. Las superficies con vegetación para interceptar la escorrentía superficial y las que permiten la infiltración de las precipitaciones tienen coeficientes de escorrentía más bajas (cercanas a 0 ). Si todos los demás factores son iguales, una zona con una mayor pendiente tendrá más escorrentía de aguas pluviales y así un coeficiente de escorrentía más alto que una zona con una pendiente inferior. Los suelos que tienen un alto contenido de arcilla no permiten mucha infiltración 
y por lo tanto tienen coeficientes relativamente altos de escurrimiento, mientras que los suelos con alto contenido de arena tienen mayores tasas de infiltración y bajos coeficientes de escorrentía.

Este coeficiente se puede cuantificar en términos de tipo de suelo, pendiente topográfica y tipo de cobertura. Numerosa bibliografía puede consultarse para estimar los coeficientes de escurrimiento (Chow et al, 1988; McCuen 1998). En el caso de que haya heterogeneidades en dichos parámetros, debe ser estimado mediante áreas de ponderación un valor representativo del coeficiente de escurrimiento. En este caso un $\mathrm{C}$ compuesto se puede definir como:

$\mathrm{C}_{\mathrm{c}}=\frac{\sum \mathrm{C}_{\mathrm{i}} \mathrm{A}_{\mathrm{i}}}{\sum \mathrm{A}_{\mathrm{i}}}$

Donde $\mathrm{C}_{\mathrm{c}}$ es el coeficiente de escorrentía compuesto, $\mathrm{C}_{\mathrm{i}}$ son los coeficientes de escorrentía de áreas homogéneas y $\mathrm{A}_{\mathrm{i}}$ son áreas individuales con $\mathrm{C}$ homogéneo. Esta información se puede estimar mediante imágenes satelitales, cartografía de suelos y modelos digitales del terreno.

La intensidad media máxima, i, se determina a partir de curvas Intensidad-Duración-Recurrencia (IDR) construidas con datos medidos en el sitio en estudio. Con dichas curvas se adopta una duración de diseño igual al tiempo de concentración de la cuenca y para una recurrencia especificada se obtiene la intensidad media máxima.

El tiempo de concentración de una cuenca, Tc, se define como el tiempo que demora el sector más alejado de la cuenca en aportar a la sección de salida. El tiempo de concentración se calcula como la suma de los tiempos de escurrimiento mantiforme (no encauzado) y el tiempo de flujo canalizado (encauzado). Pueden existir varios recorridos posibles de flujo para las diferentes áreas drenadas, el mayor tiempo de concentración de todos los tiempos, para los diferentes recorridos, es el tiempo de concentración crítico a adoptar para el área drenada.

\section{CARACTERÍSTICAS DEL ÁREA DE ESTUDIO}

Las cuencas que fueron analizadas forman parte de la cuenca del arroyo Ludueña, el que cruza el casco urbano de la ciudad de Rosario por el sector norte. Se localizan al Noroeste del municipio, sector afectado por los desbordes del arroyo y de sus afluentes, los canales Salvat e Ibarlucea.

El estudio se ha realizado en tres de las dieciocho subcuencas de la ciudad (Figura 1).

Para la selección de las mismas se tuvo en cuenta la conveniencia de analizar situaciones urbanas y periurbanas diversas, es decir, áreas no urbanizadas, áreas parcialmente urbanizadas y áreas altamente urbanizadas.



Figura 1. Subcuencas urbanas analizadas de la ciudad de Rosario.

La Subcuenca 09 (SC09) está ubicada al Noroeste del territorio Municipal. Pertenece casi en su totalidad al Distrito Norte y ocupa un área de 1641 ha. Presenta gran diversidad de usos de suelo y contiene una de las zonas no urbanizables de mayor superficie que aún se mantienen en el municipio, la cual representa alrededor del $40 \%$ del total de la subcuenca. Incluye sectores residenciales correspondientes a grupos medios, medios-bajos y bajos. Existen numerosos barrios construidos por el estado, otros están actualmente en construcción y si bien una gran parte de su superficie urbanizable no está todavía edificada, están planificados diversos proyectos de vivienda social que prevén tipologías residenciales colectivas y con uso privado del suelo. En el caso de éstas últimas, de acuerdo a las tendencias evidenciadas en la ciudad, la superficie verde prevista va edificándose progresivamente, por lo cual, a mediano plazo, presentan un alto grado de impermeabilización del 
suelo a pesar de mantener una baja densidad poblacional. El estudio de la SC09 reviste especial interés debido a que están urbanizándose áreas inundables y sectores rurales que debieran preservarse para mantener la infraestructura verde urbana.

La Subcuenca 03 (SC03) se localiza en el centroOeste de la ciudad y su borde Oeste coincide con parte del límite jurisdiccional del municipio, sobre el frente rural. Esta subcuenca es extensa, abarcando una superficie de 1952 ha, cerca del 45\% de la superficie del Distrito Municipal Noroeste, al que pertenece. Presenta sectores altamente urbanizados, barrios periurbanos de baja densidad, zonas industriales y rurales. La diversidad de usos de suelo determina una densidad media de población baja. Esta subcuenca resulta de interés por la intensiva urbanización del suelo inundable mediante las modalidades de barrios abiertos y cerrados con tipologías residenciales de baja densidad, y porque se han producido inundaciones recientes en sectores urbanizados. Estas inundaciones fueron ocasionadas por la imposibilidad de evacuación de la infraestructura pluvial en el arroyo Ludueña debida a sus crecidas súbitas, influidas por las intensas lluvias y la impactante urbanización de su vaso de inundación.

La Subcuenca 10 (SC10), se ubica en el centro-Oeste de la ciudad, al Sureste de la SC03. Tiene una superficie de 1905 ha y, a excepción del sector Sur, correspondiente al Distrito Oeste, pertenece en su totalidad al Distrito Noroeste. Integra áreas urbanizadas de diverso valor inmobiliario, áreas periurbanas y rurales. Presenta sectores forestados, hortícolas tradicionales y agrarios intensivos, además de grandes superficies vacantes. Reviste interés para la investigación ya que en ella se localizan zonas densamente urbanizadas que se han inundado recientemente que nunca antes habían sido alcanzadas por el agua y porque contiene una superficie rural significativa que constituye un componente relevante de la infraestructura verde de la ciudad. El sector Noroeste de la SC10 está siendo rápidamente ocupado por tejido residencial de baja densidad tras diversas operaciones inmobiliarias que otorgaron alto valor al suelo de este sector del vaso de inundación. Otro aspecto que debe mencionarse es que acaba de habilitarse un parque industrial de gran superficie en un área actualmente no urbanizada y con coberturas vegetales. Estos sectores debieran planificarse considerando preservar superficies permeables suficientes para evitar el agravamiento de los conflictos.

\section{APLICACIÓN DE LA METODOLOGÍA CONVENCIONAL PARA LA OBTENCIÓN DE LOS COEFICIENTES DE ESCORRENTÍA}

Para la evaluación de los coeficientes de escorrentía, la literatura proporciona tablas. En este documento, se utilizaron las tablas propuestas por Chow et al. (1988) para áreas urbanas y Mc Cuen (1998) para áreas no urbanizables. Los coeficientes de escorrentía de la cuenca dependen del uso de la tierra, el tipo de suelo y la pendiente de la cuenca. El área de drenaje de la cuenca se determinó a partir de mapas topográficos y estudios de campo. Se consideraron tres tipos de suelo, que se identificaron previamente como tipos de suelo hidrológicos B, C y $\mathrm{D}$ (ver Chow et al., 1988). Los tipos de suelo hidrológicos considerados fueron el grupo B (loess poco profundo, limo arenoso, tasa mínima de infiltración 3.8-7.6 $\mathrm{mm} / \mathrm{h}$ ), grupo C (margas arcillosas, marga arenosa poco profunda, suelos con bajo contenido orgánico, suelos generalmente altos en arcilla, tasa mínima de infiltración: $1.3-3.8 \mathrm{~mm} / \mathrm{h}$ ) y grupo D (suelos que se hinchan significativamente cuando están húmedos, arcillas pesadas, ciertos suelos salinos, tasa mínima de infiltración: 0-1.3 $\mathrm{mm} / \mathrm{h})$. La pendiente se estimó en función de la información topográfica y solo se usó una clase ya que la pendiente promedio de la cuenca es de aproximadamente $1 \mathrm{~m} / \mathrm{km}$. El coeficiente de escorrentía compuesto (ecuación 2) para varias coberturas de tierra en el área de la cuenca fue calculado con información adicional usando imágenes satelitales (Google-Earth). Esta información fue verificada in situ por los recorridos en los distintos sectores de las cuencas estudiadas.

\section{Resultados}

La metodología convencional antes detallada fue aplicada en las tres cuencas urbanas seleccionadas para el estudio. A tal efecto se propusieron 31 categorías de usos de suelo según se muestra en la Figura 2. En la Figura 3, Figura 4 y Figura 5 se muestran con códigos de color las diferentes coberturas clasificadas para las tres cuencas de estudio (Piacentini et al., 2015, Zimmermann et al., 2016, Zimmermann y Bracalenti, 2015). Las condiciones de uso de la tierra; los datos de suelos identificados para las cuencas para definir los coeficientes de escorrentía y los valores utilizados se muestran en la Tabla 1. 

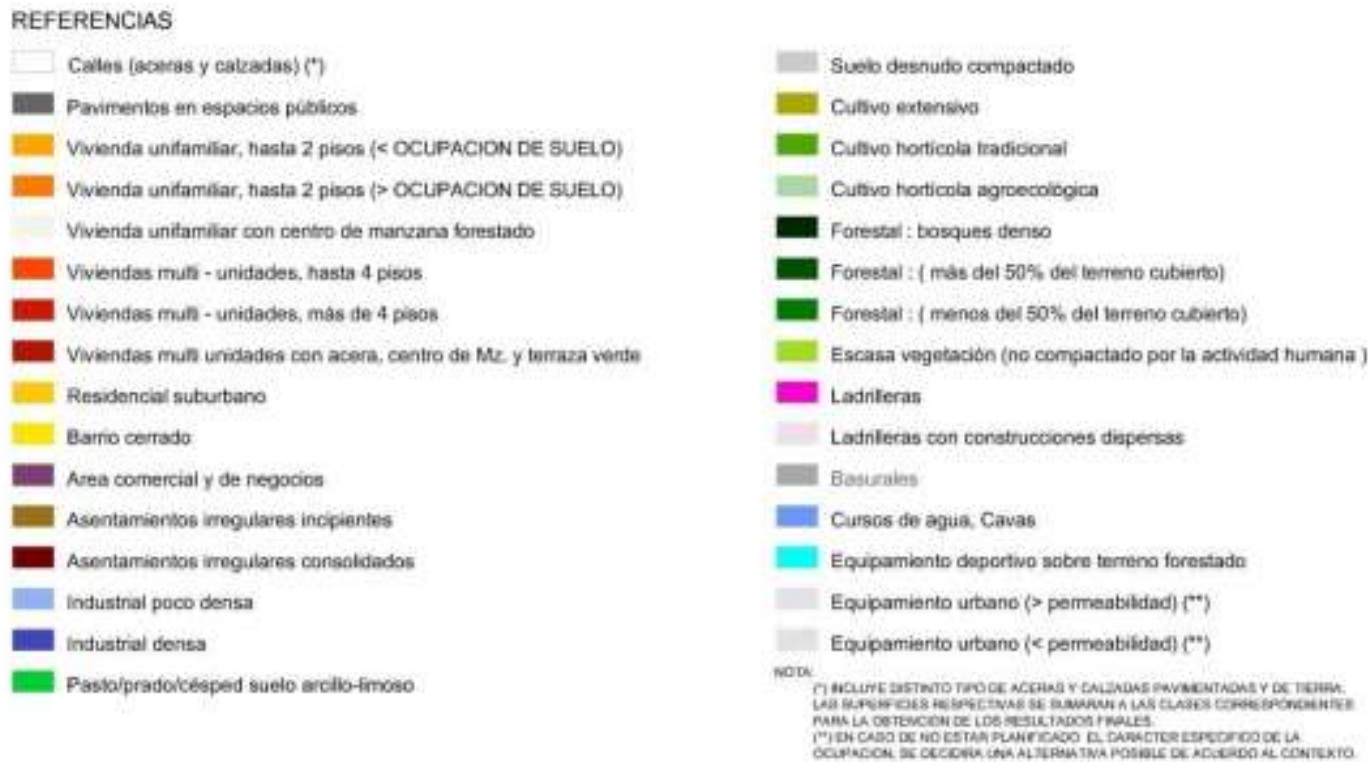

Figura 2. Referencias para los usos de suelo en las figuras.

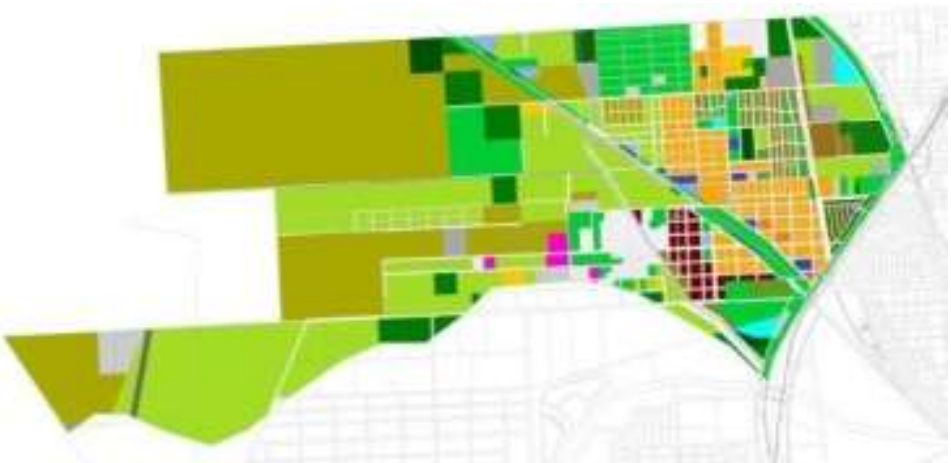

Figura 3. Usos del suelo estimados para la subcuencas 09

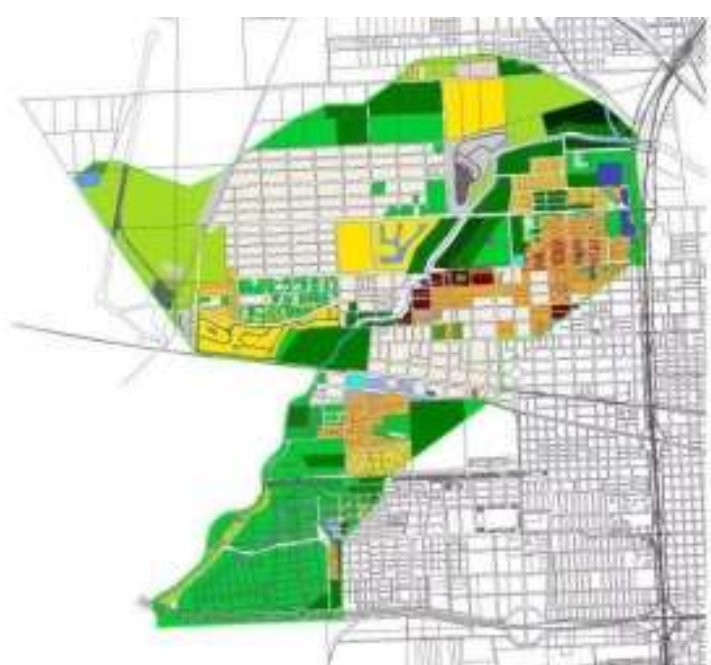

Figura 4. Usos del suelo estimados para la subcuenca 03 


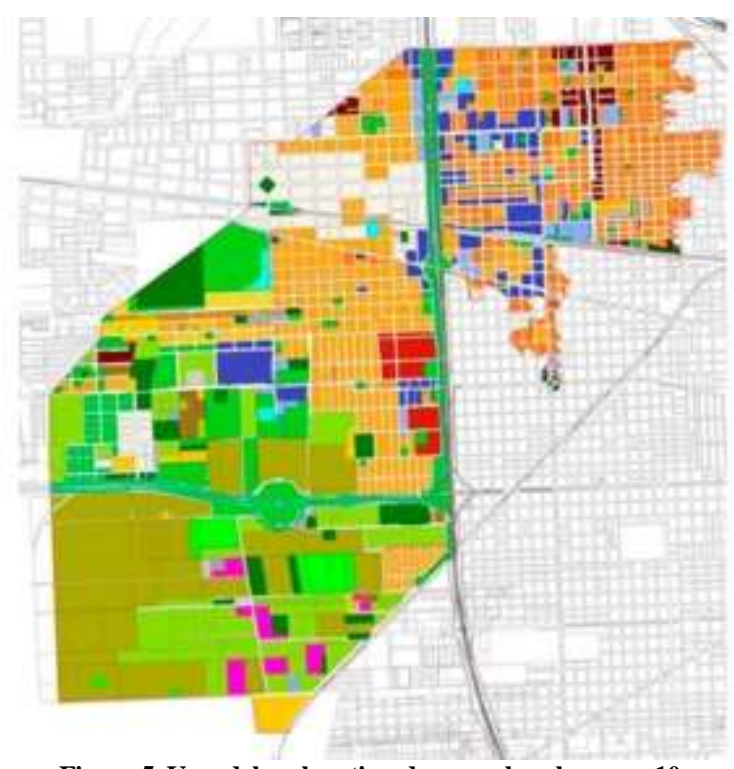

Figura 5. Usos del suelo estimados para la subcuenca 10

Tabla 1.Porcentajes de coberturas y coeficientes de escurrimientos medios para cada subcuenca estudiada

\begin{tabular}{|c|c|c|c|c|}
\hline & & SC 09 & SC 03 & SC 10 \\
\hline Coberturas & $\mathrm{C}$ & 响 Sup & \% Sup & $\%$ Sup \\
\hline \multicolumn{5}{|l|}{ Calles (aceras y calzadas) (*) } \\
\hline Pavimentos en espacios públicos & 0.90 & 3.36 & 5.90 & 16.91 \\
\hline Vivienda unifamiliar, hasta 2 pisos ( $<$ OCUPACION DE SUELO) & 0.60 & 4.68 & 4.88 & 9.12 \\
\hline Vivienda unifamiliar, hasta 2 pisos ( $>$ OCUPACION DE SUELO) & 0.65 & 0.16 & 0.09 & 6.95 \\
\hline Vivienda unifamiliar con centro de manzana forestado & 0.57 & 0.20 & 16.98 & 5.63 \\
\hline Viviendas multi - unidades, hasta 4 pisos & 0.70 & 1.05 & 0.43 & 0.02 \\
\hline Viviendas multi - unidades, mas de 4 pisos & 0.75 & - & 0.01 & 0.00 \\
\hline Viviendas multi unidades con acera, centro de Mz. y terraza verde & 0.48 & - & - & 1.50 \\
\hline Residencial o suburbano & 0.40 & 0.79 & 1.89 & 0.60 \\
\hline Barrio cerrado & 0.48 & - & 6.78 & - \\
\hline Area comercial y de negocios & 0.60 & - & - & 0.52 \\
\hline Asentamientos irregulares incipiente & 0.56 & 0.95 & 0.06 & 1.19 \\
\hline Asentamientos irregulares consolidados & 0.70 & 1.19 & 0.93 & 1.52 \\
\hline Industrial poco densa & 0.70 & 0.48 & 0.62 & 3.70 \\
\hline Industrial densa & 0.80 & 0.42 & 0.78 & 21.09 \\
\hline Pasto/prado/césped suelo arcillo-limoso & 0.30 & 11.26 & 19.71 & 4.23 \\
\hline Suelo desnudo compactado & 0.63 & 6.96 & 12.14 & 13.10 \\
\hline Cultivo extensivo & 0.21 & 30.19 & - & 1.31 \\
\hline Cultivo hortícola tradicional & 0.20 & - & 0.28 & - \\
\hline Cultivo horticola agroecológico & 0.19 & 0.01 & 0.02 & $\cdot$ \\
\hline Forestal : bosques denso & 0.13 & - & 0.81 & 0.11 \\
\hline Forestal : (más del $50 \%$ del terreno cubierto) & 0.23 & 1.42 & 1.28 & 2.96 \\
\hline Forestal : (menos del 50\% del terreno cubierto) & 0.33 & 6.52 & 13.36 & 8.05 \\
\hline Escasa vegetación (no compactado por la actividad bumana ) & 0.44 & 25.08 & 10.77 & 1.03 \\
\hline Ladrilleras & 0.63 & 0.43 & 0.02 & - \\
\hline Ladrilleras con construcciones dispersas & 0.74 & 2.23 & 0.41 & 0.13 \\
\hline Basurales & 0.63 & 1.58 & - & - \\
\hline Cursos de agua, Cavas & 1.00 & 0.44 & 1.63 & 0.32 \\
\hline Equipamiento deportivo sobre terreno forestado & 0.30 & 0.60 & 0.20 & - \\
\hline Equipamiento urbano ( $>$ perm eabilidad) & 0.55 & - & - & - \\
\hline Equipamiento urbano (< permeabilidad) & 0.60 & - & - & - \\
\hline Coeficiente de ecorrentía medio & & 0.40 & 0.49 & 0.51 \\
\hline
\end{tabular}




\section{METODOLOGÍA BASADA EN ÍNDICES DE CONSTRUCCIÓN}

Las zonas urbanizadas se componen de una cantidad heterogénea de materiales y tipologías constructivas, que sin embargo presentan una respuesta capaz de ser unificada en contraste con áreas vegetadas $\mathrm{o}$ cuerpos de agua. El comportamiento de estas superficies en el rango de Rojo (R) Infrarrojo cercano (NIR) e infrarrojo de onda corta (SWIR) permite su clara distinción utilizando índices normalizados: el NDVI (Normalized Difference Vegetation Index) resalta superficies vegetadas, mientras que el NDBI (Normalized Difference Building Index) y el UI (Urbanization Index) resaltan terrenos sobre los cuales hay construcciones. La gran capacidad de absorber energía de los cuerpos de agua genera que estas superficies se presenten siempre oscuras (valores de índice significativamente bajos). A su vez el grado de densidad de dichas construcciones presenta una correlación muy fuerte con los valores de índices de construcción (NDBI y UI), lo cual permite suponer a priori que también habrá un correlato entre dichos índices y el coeficiente de escorrentía C. Se propone analizar dichos índices analizando diferentes imágenes de la plataforma LANDSAT-8 OLI, cuya resolución espacial es suficiente (aunque no óptima) para la escala del presente trabajo, considerando que la campaña de inspección y relevamiento manual fue llevada a cabo durante el año 2016, y era requisito garantizar que el tejido urbano no ha sufrido modificaciones de gran magnitud entre la fecha de la inspección visual y la adquisición de la imagen, para que los resultados sean contrastables.

Los índices utilizados corresponden a las siguientes ecuaciones:

$\mathrm{NDVI}=\frac{\mathrm{NIR}-\mathrm{R}}{\mathrm{NIR}+\mathrm{R}}$

$\mathrm{NDBI}=\frac{\text { SWIR } 1-\mathrm{R}}{\text { SWIR } 1+\mathrm{R}}$

$\mathrm{UI}=\frac{\mathrm{SWIR} 2-\mathrm{R}}{\mathrm{SWIR} 1+\mathrm{R}}$

Donde SWIR1 corresponde a la banda entre longitudes de onda 1.566 y $1.651 \mu \mathrm{m}$, mientras que SWIR2 comprende la porción 2.107 a $2.294 \mu \mathrm{m}$. Los rangos de banda rojo y NIR son respectivamente 0.636 a 0.673 $\mu \mathrm{m}$ y $0.851-0.879 \mu \mathrm{m}$.
El procedimiento consistió en construir una relación biunívoca entre los índices normalizados de construcción y urbanización (NDBI y UI respectivamente) respecto del coeficiente de escorrentía asignado previamente mediante el procedimiento convencional. Se consideraron las áreas tipificadas en 31 clases conforme a lo previamente descrito, para luego extrapolar dicha relación al resto de la ciudad de Rosario, incluyendo las cuencas de estudio. Se analizaron 3 imágenes distintas entre 2014 y 2016, preseleccionadas bajo la condición que estuvieran libres de cobertura nubosa y no estuvieran alejadas del período de relevamiento in situ. Las fechas analizadas fueron 15 de Enero de 2014, 21 de Enero de 2016, y 13 de Agosto de 2015. Esta última corresponde a la época invernal, mientras que las primeras son de verano, para poder analizar también la incidencia estacional en las correlaciones.

A partir del trabajo simultáneo con el plano confeccionado con la metodología tradicional e imágenes de Google Earth, se identificaron parcelas homogéneas de extensión significativa para cada una de las clases (Figura 6).

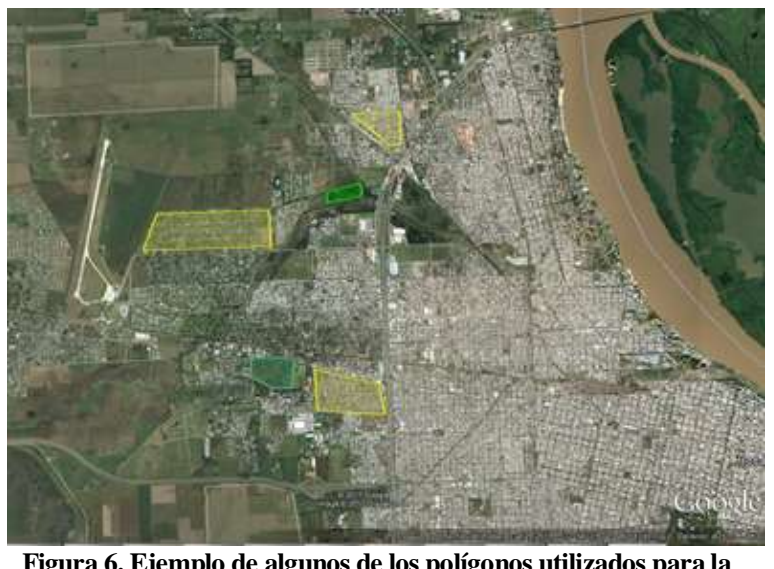

Figura 6. Ejemplo de algunos de los polígonos utilizados para la regresión lineal coeficiente $\mathbf{C}$, índice de construcción.

Se optó por reagrupar las 31 categorías originales de uso de suelo en 7 clases, ya que el nivel de resolución espacial moderada de la plataforma Landsat no admite tal nivel de detalle, cubriendo el píxel un área de 30 metros por 30 metros.

Los polígonos con cobertura homogénea obtenidos fueron superpuestos a la imagen satelital de los índices, y se extrajeron los valores representativos de cada cobertura: media aritmética, valores máximo y mínimo, considerando el 95\% de los datos, ya que se consideró que los píxeles con valores extremos podrían presentar anomalías no representativas de la clase en análisis. 
Cabe aclarar que previamente fueron enmascarados los pixeles de agua pura tal que no incidieran en desvíos dentro del análisis.

De la confrontación en un gráfico de ejes ortogonales entre los coeficientes de escorrentía de cada clase y su respectivo índice de urbanización medio se observó una fuerte correlación lineal positiva entre ambos (Figura 7).

Se realizaron ajustes en la selección de las áreas de interés a partir de la inspección visual de aquellas zonas que presentaban mayor divergencia respecto de la recta de ajuste entre ambas variables, comprobando que en todos los casos tal desvío era producto de errores en la selección original propuesta de los polígonos, al incluir píxeles que no pertenecían a la clase en cuestión y alteraban los estadísticos. Además, se incorporaron 2 clases más en correspondencia con las porciones de la trama urbana más densa dentro del microcentro y primer anillo perimetral de la ciudad ya que tal nivel de construcción no se encontraba representado en el trabajo de relevamiento antes mencionado.

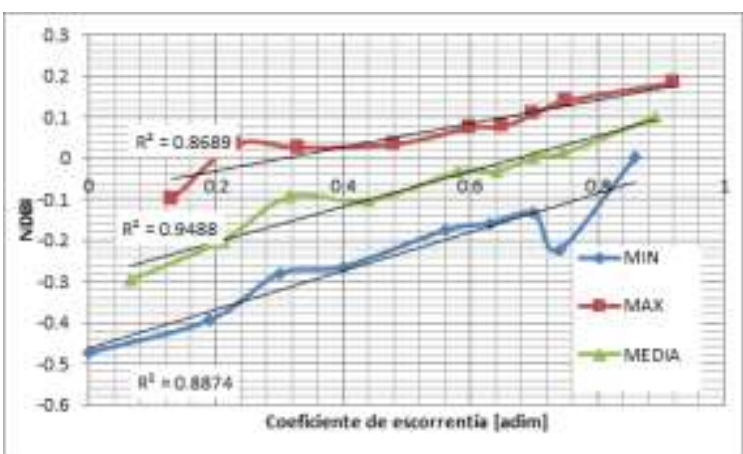

Figura 7. Relación Coeficiente de escorrentía C, índice NDBI para la imagen del 15/01/2014. Valores máximo, mínimo y media aritmética representados para cada una de las 9 clases.

Una vez completados los ajustes de las regiones de interés, y habiendo alcanzado en todos los casos una ecuación de regresión lineal con coeficiente de determinación $\mathrm{R}^{2}$ superior a 0.94 y desvíos de cada área de interés despreciables respecto de la recta de ajuste, se aplicó la ecuación de dicha recta a la imagen completa de la ciudad de Rosario y alrededores para estimar un mapa de coeficiente de escorrentía C desagregado arealmente.

La validación se realizó calculando los valores medios de cada una de las 3 subcuencas donde se había realizado el relevamiento original, respecto de la media estimada a partir de cada índice de urbanización.
El mismo procedimiento se aplicó para imágenes de índice NDVI, pero al presentar un ajuste de menor calidad fue descartado.

\section{Resultados}

Respecto de la metodología basada en índices de construcción, un primer punto sobresaliente es la fuerte correlación lineal que presentan los índices UI y NDBI con el coeficiente de escorrentía siendo éstas cercanas a la unidad, lo cual permitió suponer que el mapa desagregado de coeficiente $\mathrm{C}$ presentaría un nivel de precisión y ajuste a destacar.

Se observa en la Figura 8 que a medida que aumenta el grado de impermeabilización de la cobertura $(\mathrm{C}$ tiende a 1), la relación entre el coeficiente de escorrentía y el índice NDBI tiende a ser lineal. Es posible suponer que los desvíos en las áreas de coeficiente bajo respecto de dicha recta se deban a distorsiones introducidas por sectores de la superficie que no presenten cobertura vegetal frondosa.

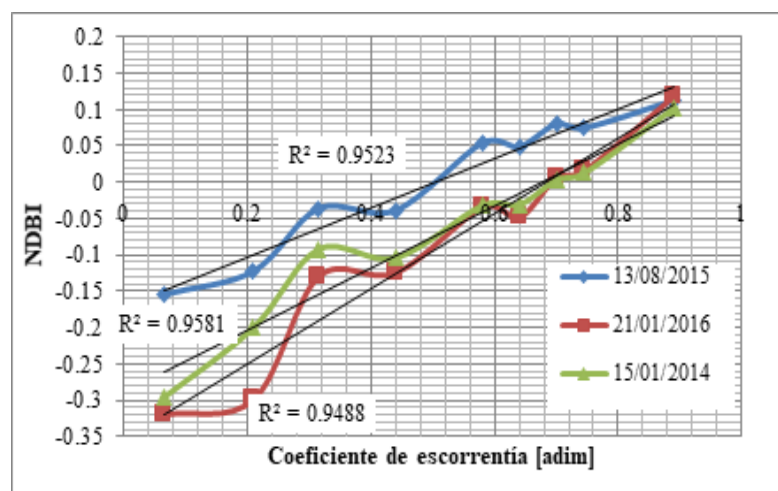

Figura 8. Recta correlación Coeficiente escorrentía, índice NDBI, para cada fecha analizada.

No obstante estos resultados fueron alcanzados luego de una serie de ajustes en las regiones homogéneas a partir del proceso iterativo de propuesta y revisión de las áreas cuyos valores medios presentaran una diferencia significativa respecto de la recta de regresión. Esto permite apreciar la sensibilidad del método a la correcta selección de tales áreas.

Cabe aclarar que el procedimiento se repitió para cada escena y se construyó una ley de correlación específica por fecha.

Asimismo la imagen de invierno precisó la identificación de nuevas áreas de interés en la zona rural, debido a que presentaba valores de índice muy 
elevados producto de la alta reflectancia del suelo desnudo en la combinación de bandas consideradas.

Se realizaron estimaciones del coeficiente $\mathrm{C}$ a partir de la ecuación de la recta que lo relaciona con el índice NDBI para cada fecha.

La comparación final se realizó a partir del contraste entre el coeficiente de escorrentía medio asignado a cada subcuenca, respecto del estimado con la metodología antes descripta. En la Tabla 2se presenta un resumen de los resultados por fecha y por índice de construcción.

Tabla 2. Coeficiente de escorrentía asignado por relevamiento y estimado con índices de construcción para cada fecha. (a) NDBI, (b) UI.

(a) NDBI.

\begin{tabular}{|c|c|c|c|c|}
\hline Subcca & In situ & $\mathbf{1 5 / 0 1 / 1 4}$ & $\mathbf{1 3 / 0 8 / 1 5}$ & $\mathbf{2 1 / 0 1 / 1 6}$ \\
\hline 3 & 0.49 & 0.45 & 0.51 & 0.45 \\
\hline 9 & 0.4 & 0.44 & 0.63 & 0.42 \\
\hline 10 & 0.52 & 0.50 & 0.63 & 0.47 \\
\hline
\end{tabular}

(b) UI.

\begin{tabular}{|c|c|c|l|c|}
\hline Subcca & In situ & $\mathbf{1 5 / 0 1 / 1 4}$ & $\mathbf{1 3 / 0 8 / 1 5}$ & $\mathbf{2 1 / 0 1 / 1 6}$ \\
\hline 3 & 0.49 & 0.41 & 0.46 & 0.43 \\
\hline 9 & 0.4 & 0.39 & 0.54 & 0.39 \\
\hline 10 & 0.52 & 0.51 & 0.63 & 0.49 \\
\hline
\end{tabular}

Los resultados obtenidos son altamente satisfactorios, y las estimaciones realizadas con ambos índices de construcción se ajustan a los valores asignados a partir del relevamiento original. Esto pondera positivamente la metodología propuesta, aunque cabe realizar la salvedad de que las escenas de verano presentan mayor precisión, tal como era de esperar ya que la vegetación presente dentro de la trama urbana y en la zona periurbana es más frondosa y cubre mejor el suelo subyacente, el cual podría ser fuente de incertidumbre en las estimaciones.

Por otro lado no es posible concluir que uno de los índices de construcción tenga un comportamiento mejor que el otro. Puede observarse que la cuenca con mayor índice de vegetación NDVI (subcuenca 9) es aquella que presenta los desvíos más importantes en ambos casos. Esto permite proponer como línea de investigación futura la incorporación y combinación de información de cobertura vegetal con los índices de construcción.
Asimismo las gráficas de caja de la Figura 9 sintetizan algunas particularidades observadas para las distintas alternativas exploradas, siendo la recta horizontal la media asignada para cada subcuenca según lo observado en terreno.

En primer lugar se puede observar que no hay mayores diferencias entre las estimaciones con cada índice de construcción, aunque sí hay un mayor rango de valores de $\mathrm{C}$ entre el primer y tercer cuartil.

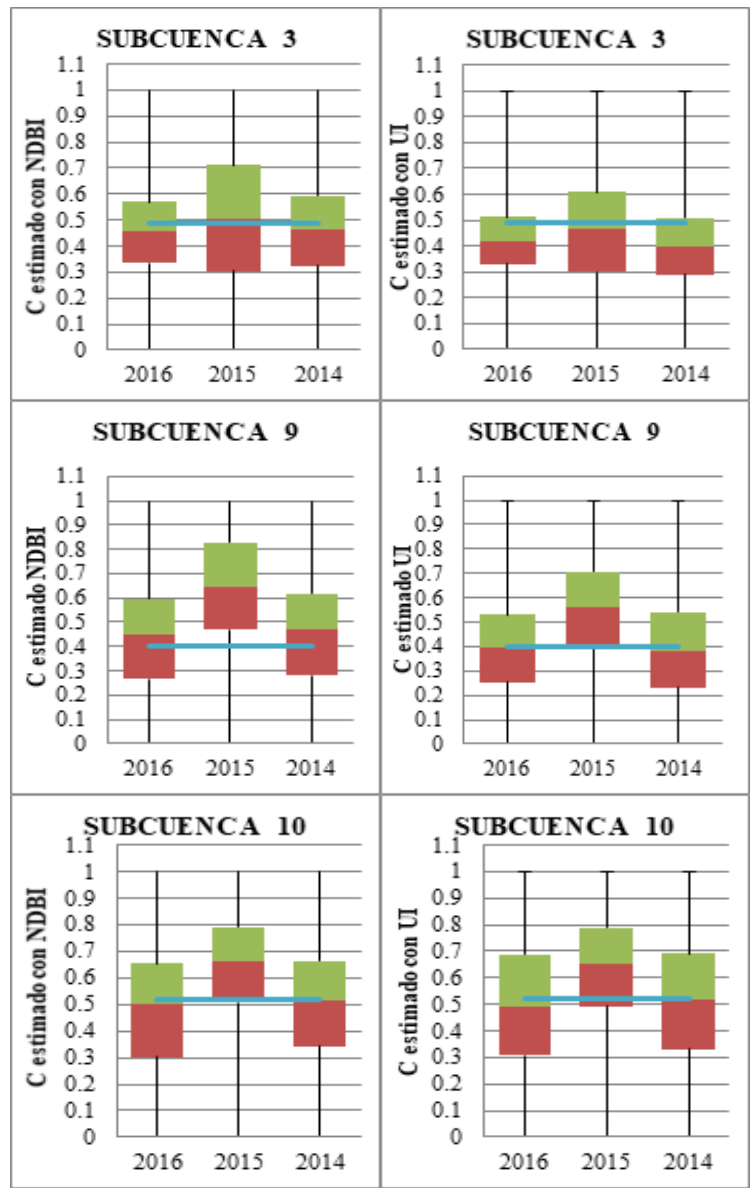

Figura 9. Gráficos de cajas diferenciados por subcuenca, para cada fecha analizada. La columna izquierda presenta los valores de $\mathrm{C}$ estimados con NDBI, y la de la derecha los que corresponden a UI.

Se destaca también la sobreestimación de la escena de invierno, especialmente para las subcuencas 9 y 10, que son precisamente aquellas que mejor ajuste presentan para las escenas de verano.

Se pretendió justificar estos fenómenos a partir de la identificación de mayores porciones de terreno vegetado, no obstante no se verificó esto en los valores medios de NDVI por zona, sino que los valores 
observados en cada escena tienen divergencias despreciables entre subcuencas, aunque se puede apreciar que la 9 presenta mayor superficie con espacios verdes.

\section{METODOLOGÍA BASADA EN CLASIFICACIÓN SUPERVISADA IMÁGENES SATELITALES}

LA

DE

Con el objeto de encontrar una metodología que permita estimar de manera sistematizada valores de coeficientes de escorrentía de diferentes subcuencas, en este segundo caso se utilizó, dentro de las técnicas de teledetección, la clasificación supervisada de una imagen satelital para determinar distintos tipos de cobertura o usos de suelo presentes en la región en estudio que puedan correlacionarse con los mismos.

Partiendo de la base que cada material posee una determinada "firma espectral", se puede asociar a cada pixel de una imagen satelital un determinado uso del suelo.

Con esta metodología se pudo clasificar la imagen en 7 tipos de cobertura a saber: suelo desnudo, cultivos, césped, hormigón, pavimento asfáltico y chapa. Cada tipología queda entonces identificada como una clase diferente. Luego, a cada uno de estos tipos de cobertura se le asignaron coeficientes de escorrentía asociados para determinar un $\mathrm{C}$ compuesto en cada subcuenca estudiada mediante la ecuación 2.

Teniendo en cuenta los resultados obtenidos mediante la metodología basada en el índice de construcción, se decidió utilizar la imagen del 21 de Enero de 2016 tomada por el satélite LANDSAT 8, que fue con la que se obtuvieron mejores ajustes.

De todas las bandas disponibles en las imágenes de LANDSAT 8, se utilizaron para el procesamiento sólo 6 , las tres del espectro visible (rojo, verde y azul) y otras tres del rango no visible (una del infrarrojo cercano y dos del infrarrojo medio).

Se realizó una clasificación supervisada de la imagen definiendo previamente áreas de entrenamiento o regiones de interés (ROIs) según los distintos tipos de cobertura. A partir de estas regiones se calcularon las estadísticas para clasificar a cada pixel de la imagen dentro de una categoría según su probabilidad de pertenecer a la misma. En este caso se utilizó el método de la Máxima Verosimilitud, por haber comprobado previamente que se obtienen mejores resultados para este tipo de clasificaciones (Riccardi et al., 2018).

En la determinación de los sitios de entrenamiento se tuvo la precaución de buscar píxeles en "regiones puras" según el tipo de cobertura, de manera que no exista mucha dispersión en las firmas espectrales de cada tipo, ya que esto luego introduce errores en la clasificación de la imagen. Esta tarea no es dificultosa para los tipos de cobertura que se dan en la zona rural (suelo desnudo, cultivado, césped, etc.), pero en la zona más urbanizada esto se complejiza teniendo en cuenta que el tamaño de las construcciones en general es menor al tamaño del píxel.

Una vez determinadas las regiones de entrenamiento, se enmascaró la zona de islas y el río Paraná, quedando como resultado una imagen como la que se visualiza en la Figura 10. En la misma se representaron en color blanco todos los píxeles clasificados como superficie impermeable (hormigón, chapa y asfalto) y en marrón y distintos tonos de verde, los correspondientes a superficies permeables (suelo desnudo, césped, cultivos y arboleda respectivamente).

La metodología de clasificación supervisada fue validada en varios trabajos previos (Mendez Zacarías y Zimmermann, 2011; Mendez Zacarías y Zimmermann, 2014 y Riccardi et al., 2018).

Finalmente, se puede asociar cada uno de los tipos de suelo identificados en la clasificación supervisada a un coeficiente de escorrentía en función de sus características, para luego estimar valores medios de C de cada una de las cuencas en estudio. En la Tabla 3 se presentan los tipos de cobertura de suelo y el coeficiente $\mathrm{C}$ asignado a cada uno.

Tabla 3. Coeficiente de escorrentía para los distintos tipos de cobertura.

\begin{tabular}{|c|c|}
\hline Cobertura suelo & C \\
\hline césped & 0.3 \\
\hline chapa & 0.9 \\
\hline asfalto & 0.9 \\
\hline hormigón & 0.9 \\
\hline arboleda & 0.23 \\
\hline cultivo & 0.2 \\
\hline suelo desnudo & 0.44 \\
\hline
\end{tabular}






Figura 10. Clasificación supervisada imagen satelital.

\section{Resultados}

Respecto a la metodología desarrollada a partir de la clasificación supervisada, se determinaron valores medios de coeficientes de escorrentía para cada una de las cuencas en estudio a partir de la Ecuación 2.

En la Tabla 4, Tabla 5 y Tabla 6 se presentan los resultados obtenidos en cada subcuenca.

Tabla 4.Coeficiente de escorrentía ponderado Subcuenca 03.

\begin{tabular}{|c|c|c|c|c|}
\hline \multicolumn{5}{|c|}{ Arroyo Ludueña. } \\
\hline $\begin{array}{c}\text { Tipo de } \\
\text { cobertura }\end{array}$ & $\mathbf{C}$ & $\begin{array}{c}\text { Cant. } \\
\text { Píxeles }\end{array}$ & $\begin{array}{l}\text { Porc. } \\
\text { Área }\end{array}$ & $\begin{array}{c}\text { C } \\
\text { Pond. }\end{array}$ \\
\hline Césped & 0.3 & 14533 & $67 \%$ & 0.20 \\
\hline $\begin{array}{c}\text { Chapa/ } \\
\text { Asfalto/ } / \mathrm{H}^{\circ}\end{array}$ & 0.9 & 3186 & $15 \%$ & 0.13 \\
\hline Arboleda & 0.23 & 801 & $4 \%$ & 0.01 \\
\hline Cultivo & 0.2 & 69 & $<1 \%$ & $<0.01$ \\
\hline \multirow[t]{2}{*}{$\begin{array}{c}\text { Suelo } \\
\text { desnudo }\end{array}$} & 0.44 & 3083 & $14 \%$ & 0.06 \\
\hline & Total & 21672 & $100 \%$ & 0.41 \\
\hline
\end{tabular}

Tabla 5.Coeficiente de escorrentía ponderado Subcuenca 09.

\begin{tabular}{|c|c|c|c|c|}
\hline \multicolumn{5}{|c|}{ Canal Ibarlucea. } \\
\hline $\begin{array}{c}\text { Tipo de } \\
\text { cobertura }\end{array}$ & $\mathbf{C}$ & $\begin{array}{c}\text { Cant. } \\
\text { Píxeles }\end{array}$ & $\begin{array}{l}\text { Porc. } \\
\text { Área }\end{array}$ & $\begin{array}{c}\text { C } \\
\text { Pond. }\end{array}$ \\
\hline Césped & 0.3 & 12230 & $64 \%$ & 0.19 \\
\hline $\begin{array}{c}\text { Chapa/ } \\
\text { Asfalto/H } \mathrm{H}^{\circ}\end{array}$ & 0.9 & 2147 & $11 \%$ & 0.10 \\
\hline Arboleda & 0.23 & 750 & $4 \%$ & 0.01 \\
\hline Cultivo & 0.2 & 1086 & $7 \%$ & 0.01 \\
\hline \multirow[t]{2}{*}{$\begin{array}{c}\text { Suelo } \\
\text { desnudo }\end{array}$} & 0.44 & 2887 & $15 \%$ & 0.07 \\
\hline & Total & 19100 & $100 \%$ & 0.38 \\
\hline
\end{tabular}

Tabla 6. Coeficiente de escorrentía ponderado Subcuenca 10.

\begin{tabular}{|c|c|c|c|c|}
\hline $\begin{array}{c}\text { Tipo de } \\
\text { cobertura }\end{array}$ & C & $\begin{array}{c}\text { Cant. } \\
\text { Píxeles }\end{array}$ & $\begin{array}{c}\text { Porc. } \\
\text { Área }\end{array}$ & $\begin{array}{c}\text { C } \\
\text { Pond. }\end{array}$ \\
\hline Césped & 0.3 & 19466 & $46 \%$ & 0.14 \\
\hline $\begin{array}{c}\text { Chapa/ } \\
\text { Asfalto/H }\end{array}$ & 0.9 & 15758 & $37 \%$ & 0.34 \\
\hline Arboleda & 0.23 & 1392 & $3 \%$ & 0.01 \\
\hline Cultivo & 0.2 & 1316 & $3 \%$ & 0.01 \\
\hline $\begin{array}{c}\text { Suelo } \\
\text { desnudo }\end{array}$ & 0.44 & 4442 & $11 \%$ & 0.05 \\
\hline & Total & $\mathbf{4 2 3 7 4}$ & $\mathbf{1 0 0 \%}$ & $\mathbf{0 . 5 3}$ \\
\hline
\end{tabular}

\section{COMPARACIÓN METODOLOGÍAS}

ENTRE

LAS

Finalmente se compararon los resultados obtenidos mediante las tres metodologías planteadas. Los mismos se presentan en la Tabla 7.

Tabla 7. Comparación entre las distintas metodologías.

\begin{tabular}{|c|c|c|c|c|}
\hline \multirow{2}{*}{ Cuenca } & \multicolumn{4}{|c|}{ C Estimados } \\
\cline { 2 - 5 } & Conv. & NDBI & UI & Clasif. Sup. \\
\hline SC 03 & 0.49 & 0.45 & 0.43 & 0.41 \\
\hline SC 09 & 0.40 & 0.42 & 0.39 & 0.38 \\
\hline SC 10 & 0.51 & 0.47 & 0.49 & 0.53 \\
\hline
\end{tabular}

Se observa que la máxima diferencia respecto a la metodología convencional utilizada como referencia, es del orden del $16 \%$ y se da para la Subcuenca SC 03. Esta diferencia se observa en el caso de la clasificación supervisada. La subcuenca SC 03 presenta una tipología de vivienda característica de barrios abiertos, donde los espacios permeables e impermeables configuran un tejido más heterogéneo que en las otras subcuencas, por ello la determinación del tipo de cobertura es más dificultosa.

En el resto de los casos las diferencias se acotan en un rango entre el $3 \%$ y $12 \%$, validando las metodologías presentadas.

Los métodos dan diferencias tanto por exceso como por defecto y ninguno presenta un sesgo en particular por diferencias positivas o negativas.

\section{CONCLUSIONES}

Quedan validadas ambas metodologías para la 
estimación de valores de coeficiente de escorrentía a escala de subcuenca urbana, en zonas de baja pendiente, y con herramientas de teledetección combinadas con información de terreno. Las diferencias observadas son de poca implicancia, por lo que es posible asumir que mientras los usos de suelo en la zona de estudio no diverjan significativamente de los observados en este trabajo, es posible aplicar cualquiera de las metodologías propuestas.

Sin embargo, en casos donde la heterogeneidad de los usos de suelo sea creciente, la segunda metodología pierde sensibilidad y podría tender a evidenciar errores mayores.

El nivel de detalles en la cobertura y en consecuencia el coeficiente de escorrentía estimado, está vinculado a resolución espacial de los sensores utilizados.

Teniendo un conocimiento previo de la zona en estudio, las metodogías basadas en teledetección permiten la estimación de los coeficientes $\mathrm{C}$ mediante el auxilio de imágenes satelitales de mayor resolución como las disponibles en Google Earth, prescindiendo de las exhaustivas tareas de campo del método convencional.

Cabe destacar que la metodología convencional demanda un minucioso detalle del tejido urbano realizado artesanalmente, lo cual demanda un importante consumo de tiempo en gabinete y en campo. En este sentido las metodologías alternativas presentan una atractiva propuesta que agiliza la estimación.

\section{REFERENCIAS}

Chow, V T., Maidment, D., Mays, L. (1988). Applied Hydrology. McGraw -Hill.

McCuen R. H. (1998). Hydrology: Analysis and Design. Prentice-Hall. 2ed.

Mendez Zacarías J. y Zimmermann E. (2011). Uso de Sistemas de Información Geográfica para parametrización de modelos de simulación hidrológica en llanuras. Congreso Nacional del Agua 2011. Resistencia. Chaco.

Mendez Zacarías, J. y Zimmermann E. (2014). Calibración del modelo SHALL con elevado nivel de detalle en la cuenca del $\mathrm{A}^{\circ}$ Ludueña utilizando SIG.
II Congreso Internacional de Hidrología de llanuras 2014. Santa Fe. Santa Fe

Piacentini, R., Feldman, S., Coronel, A., Feldman, N., Vega, M., Moskat, V., Bracalenti, L., Zimmermann, E., Lattuca, A., Biasatti, N., Dubbeling, M. (2015). Agricultura urbana y periurbana y forestación como posibilidad de mitigación y adaptación al cambio climático. Estudio de caso en la ciudad de Rosario y región, Argentina. Cambio climático. Lecciones de y para ciudades de América Latina. Sylvie Nail (ed.) Universidad Externado de Colombia. 327-368.

Riccardi, G., Mendez Zacarías, J., Odicini, L., Ramaciotti, G., Gigante Rodríguez, F., Marrone, L. (2018) Modelación hidrológica e hidráulica en las cuencas de los Emisarios Pluviales 9 Y 10 del distrito Rosario (SF). XII Jornadas de Ciencia y Tecnología 2018. Secretaría de Ciencia y Técnica. Universidad Nacional de Rosario.

Zimmermann, E. y Bracalenti, L. (2015). Reducción de riesgo de inundación urbana mediante incremento de áreas ocupadas por agricultura y forestación urbana y periurbana. El caso Rosario. Informe Proyecto: Field testing of the monitoring framework, establishing monitoring baseline, design of scenarios and policy uptake in cities. Phase 2:" Climate Development Knowledge Network (CDKN) RUAF Foundation; UN Habitat. 2012-2014.

Zimmermann, E., Bracalenti, L., Piacentini, R. e Inostroza, L. (2016). Urban flood risk reduction by increasing green areas for adaptation to climate change. Procedia Engineering and World Multidisciplinary Civil EngineeringArchitecture-Urban Planning Symposium. Edit. Elsevier. (In press).

\section{REGISTRO BIBLIOGRÁFICO:}

Chiarito, E.; Zimmermann, E. y Méndez Zacarías, S. (2018). Metodologías para la estimación del coeficiente de escorrentía en áreas urbanizadas mediante teledetección. Cuadernos del CURIHAM. 24, 25-36. DOI: 10.35305/curiham.v24i0.126

\section{Tipo de Publicación: ARTíCULO.}

Trabajo recibido el 17/09/2018 y aprobado para su publicación el 03/12/2018. 